\title{
CAE Environment for Electromechanical Microsystems
}

\author{
R.Lerch, M. Kaltenbacher, H. Landes \\ Department for Sensor Technology \\ University of Erlangen-Nuremberg \\ D-91058 Erlangen
}

\section{INTRODUCTION}

Short product lifetime cycles, fast time to market and cost reduction as well as an increasing technical complexity are only some of the challenges developers of electromechanical microsystems are faced with. Since the fabrication of prototypes and experimental based design is a lengthy and costly process, the urgent need for appropriate Computer Aided Enginecring (CAE) tools, especially numerical simulation codes, arises. Therefore, we have developed a computer aided engineering software environment for the design of microsystems containing electromechanical sensors and actuators. In this environment we are able to precisely model nearly any type of piczoelectric, clectrostatic or electromagnetic transducer including an eventual coupling to a surrounding medium, either a gas, a fluid or a solid. The software even allows the modeling of major nonlinear effects arising in electromechanical transduccrs, such as dependence of the elastic moduli on deformation, the nonlinear magnetization curves of ferroelectric materials or the geometric nonlinearities duc to large displacements.

This software scheme is called CAPA [1] and is based on finite elements (FE), boundary clements (BE) or a coupling of both (FE/BE). Several interfaces for the import or export of data to commercial CAD and numerical modeling packages, like CATIA. IDEAS, ANSYS, have also been developed. Furthermore, interfaces to circuit simulation software are provided (Fig. 1). For the numerical modeling of $\mathrm{e}$ ectromechanical sensors and actuators one has to consider the mutual interaction of different physical fields:

- Coupling Electric Field Mechanical Fïeld

This coupling is cither based on the electrostrictive or piezoclectric effect or results from the force on an electric charge in an electric field (clectrostatic force).

- Coupling Magnetic Field Mechanical Field

This coupling is twofold. We first have the clectromotive force $(\mathrm{cmf})$ which describes the generation of an electric field (electric voltage resp. current) when a conductor is moved in a magnetic field and. second, the electromagnetic force.

- Coupling Mechanical Field - Acoustic Field

Very often an electromechanical transducer is surrounded by a fluid or a gaseous medium

The original version of this chapter was revised: The copyright line was incorrect. This has been corrected. The Erratum to this chapter is available at DOI: 10.1007/978-0-387-35498-9_57 
in which an acoustic wave is launched (actuator) or is impinging from an outside source towards the receiving transducer.

In the following sections, the basic equations as well as their finite or boundary element implementations are described for these major transducing mechanisms. In each section we introduce one or more practical applications, too.

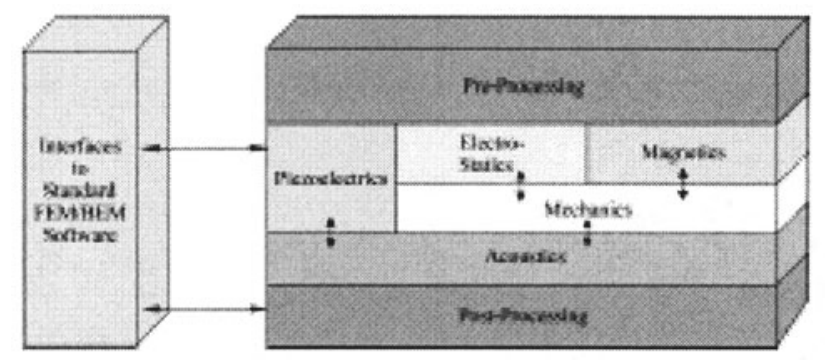

Figure 1 Software System CAPA

\section{PIEZOELECTRIC TRANSDUCERS}

\subsection{BASIC EQUATIONS}

The development of piezoelectric finite elements is based on 3 equations, namely the material equations, Newton's law and the potential equation. The material equations, which are the basis of linear piczoclectricity, take into account the piczoelectric effect both in the description of the mechanical stresses as well as the dielectric displacement. According to [2]. these equations may be written as

$$
\begin{aligned}
& \vec{T}=\mathbf{c}^{E} \vec{S}-\mathbf{e}^{T} \vec{E} \\
& \vec{D}=\mathbf{e} \vec{S}+\varepsilon^{S} \vec{E}
\end{aligned}
$$

Herein. $\vec{T}$ denotes the mechanical stress, $\mathrm{c}^{E}$ the mechanical material tensor at constant electric ficld $\vec{E}$. e the piezoelectric coupling tensor, $\varepsilon^{S}$ the dielectric tensor at constant strain $\vec{S}$ and $\vec{D}$ the electrical displacement.

Newton's law, which describes the elastic behaviour of a finite, deformable body can be expressed by the partial differential equation

$$
\begin{aligned}
\left(\frac{\partial T_{1}}{\partial x}+\frac{\partial T_{1}}{\partial y}+\frac{\partial T_{0}}{\partial z}\right) \overrightarrow{e_{1}}+ & +\left(\frac{\partial T_{2}}{\partial y}+\frac{\partial T_{1}}{\partial x}+\frac{\partial T_{5}}{\partial z}\right) \overrightarrow{e_{2}} \\
& +\left(\frac{\partial T_{3}}{\partial z}+\frac{\partial T_{5}}{\partial y}+\frac{\partial T_{6}}{\partial x}\right) \overrightarrow{e_{3}}=\rho \frac{\partial^{2} \vec{u}}{\partial t^{2}}
\end{aligned}
$$

Herein, $\rho$ denotes the density of the material and $\overrightarrow{e_{1}}, \overrightarrow{e_{2}}$ and $\overrightarrow{e_{3}}$ are unit vectors in the $x-, y-$ and $z$-direction. respectively.

Using the relation between the electric field $\vec{E}$ and the scalar electric potential $\Phi$ according to

$$
\vec{E}=-\nabla \Phi
$$


the constituitive equation for the electric field results in

$$
\Gamma \cdot \varepsilon \Gamma \Phi=q
$$

where $q$ represents the free electric volume charge.

\subsection{FINITE ELEMENT FORMULATION}

Combining (1.1)-(1.5) we get a full description of the dynamic behavior of a piezoelectric body.

The application of a finite element discretization scheme to these equations ends up with a linear system of equations, which can be summarized as follows $[3,4]$ :

$$
\begin{aligned}
\left(\begin{array}{cc}
\mathbf{M}_{u u} & 0 \\
0 & 0
\end{array}\right)\left(\begin{array}{l}
\{\ddot{u}\} \\
\{\ddot{\Phi}\}
\end{array}\right)+ & \left(\begin{array}{cc}
\mathbf{C}_{u u} & 0 \\
0 & 0
\end{array}\right)\left(\begin{array}{c}
\{\dot{u}\} \\
\{\dot{\Phi}\}
\end{array}\right) \\
& +\left(\begin{array}{cc}
\mathbf{K}_{u u} & \mathbf{K}_{u \varphi} \\
\mathbf{K}_{u \phi}^{\prime \prime} & \mathbf{K}_{\circlearrowleft \dot{\varphi}}
\end{array}\right)\left(\begin{array}{l}
\{u\} \\
\{\Phi\}
\end{array}\right)=\left(\begin{array}{c}
\{F\} \\
\{Q\}
\end{array}\right)
\end{aligned}
$$

Herein $\mathbf{K}_{u u}, \mathbf{C}_{u u}$ and $\mathbf{M}_{u u}$ denote the mechanical stiffness, damping and mass matrix, respectively, $\mathbf{K}_{\phi \phi}$ and $\mathbf{K}_{u \varphi}$ the dielectric stiffness- and the piezoelectric coupling matrix, $\{F\}$ and $\{Q\}$ the external mechanical forces and electric charges, $\{u\}$ the nodal vector of displacement and $\{\Phi\}$ the nodal vector of scalar electric potential.

Starting with the semidiscrete formulation time stepping procedures based on the Newmark or Hilber-Hughes-Taylor method [5] may be applied. Furthermore. standard algorithms for cigenvalue calculations, like the subspace and Lanczos procedures, as well as harmonic excitations are easily extended.

\subsection{ULTRASOUND ARRAYS}

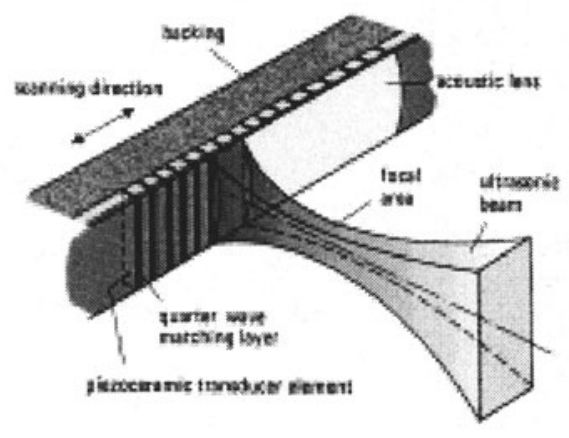

Figure 2 Ultrasound phased array antenna

Ultrasound arrays have found a wide area of applications, ranging from medical imaging to non-destructive testing. The principle of a standard ultrasound phased array antenna, as used in medical imaging applications, is shown in Fig. 2. The backing is supplied on the backside of the transducer to damp out the vibrations of the antenna and thus generate short ultrasound pulses. The lense on top of the antenna is responsible for the geometric focussing of the 
ultrasound beam whereas the matching layer(s) are used to adjust the high acoustic impedance of the piezoelectric transducer to the relatively low impedance of the lense material. Finite element simulations for such antennas have already been reported elsewhere $[3,4.6]$.

In recent times, concepts for $2 \mathrm{D}$ ultrasound arrays, in which the geometric focussing of the lense is replaced by an addititional subdicing of the array in the length direction, have been developed. $A$ new type of such a 2D ultrasound array has been developed utilizing a new silicon CMOS chip technology [7]. These transducers work simultaneously as ultrasound sensors and as ultrasound transmitters. The transducing mechanism is either piezoclectric or clectrostatic. In the piezoelectric case, a thin layer of piczoccramic material is put on top of a micromachined silicon membrane defining a bimorph transducer, while in the clectrostatic case, a voltage is applied between this membrane and a base plate, defining a capacitive transmitter (Fig. 3). Detailed results for these devices are reported in [8]

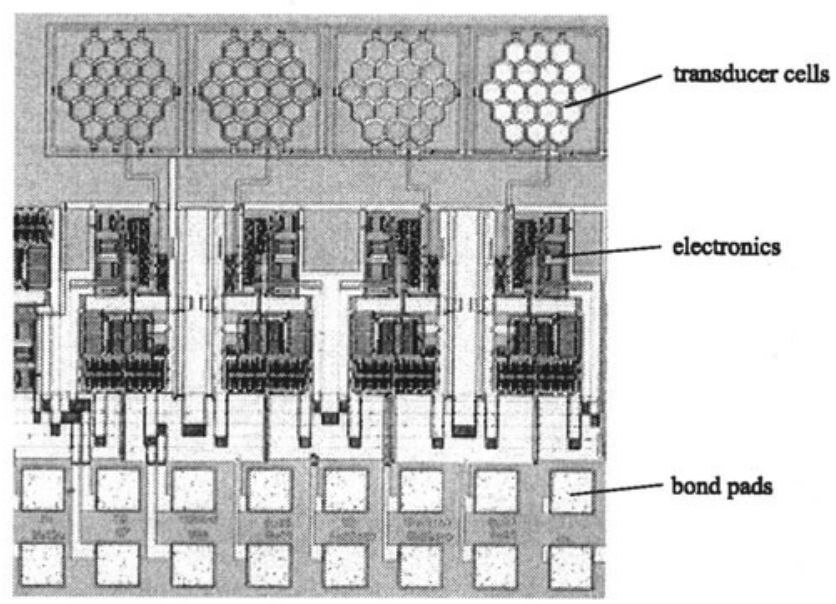

Fïgure 3 Top view of a CMOS chip with 4 areas, each containing 19 capacitive transducer cells

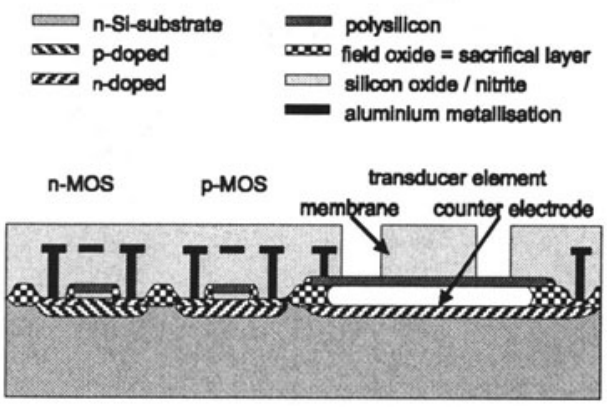

Figure 4 Schematic cross section of an electrostatic ultrasound transducer in CMOS technology 


\section{ELECTROSTATIC-MECHANICAL- TRANSDUCERS}

\subsection{BASIC EQUATIONS}

In the case of an electrostatic-mechanical-transducer the coupling between the electric and the mechanical field is caused by the electrostatic force between the electrodes. This force is calculated based on the electrostatic force tensor $\mathbf{T}_{E^{\prime}}$, where $\vec{E}=\left(E_{x}, E_{y}, E_{z}\right)$ denotes the clectric ficld

$$
\mathbf{T}_{E}=\left[\begin{array}{ccc}
\varepsilon E_{x}^{2}-\frac{1}{2} \varepsilon|E|^{2} & \varepsilon E_{z} E_{y} & \varepsilon E_{x} E_{z} \\
\varepsilon E_{y} E_{x} & \varepsilon E_{y}^{2}-\frac{1}{2} \hat{\varepsilon}|E|^{2} & \varepsilon E_{y} E_{z} \\
\varepsilon E_{z} E_{x} & \varepsilon E_{z} E_{y} & \varepsilon E_{i}^{2}-\frac{1}{2} \varepsilon|E|^{2}
\end{array}\right] .
$$

The electrostatic force $\vec{F}_{E}$ is given by

$$
\vec{F}_{L^{\prime}}=\iint_{A} \mathbf{T}_{E} \vec{n} d S
$$

where $\vec{n}$ is the normal vector on the surface $A$. The electrostatic force leads to a deformation of the electrodes, which is described by (1.3) and, therefore, introduces a geometric nonlinearity in (1.5).

\subsection{FEM-BEM-FORMULATION}

The boundary element discretization of (1.5) yields to the following BE-matrix equation

$$
\mathbf{H}_{\phi}\{\Phi\}-\mathbf{G}_{\phi}\left\{E_{n}\right\}=\{Q\}
$$

with the two boundary element matrices $\mathbf{H}_{\phi}$ and $\mathbf{G}_{\phi}$, the nodal vector of electric charge $\{Q\}$. the nodal vector $\{\Phi\}$ of the scalar electric potential and the nodal vector $\left\{E_{n}\right\}$ of the normal component of the electric field.

Applying the lE-formulation to (1.3) leads to the wellknown matrix equation for the mechanical quantitics

$$
\mathbf{M}\{\ddot{u}\}+\mathbf{C}\{\dot{u}\}+\mathbf{K}\{u\}-\left\{F\left(\Phi, E_{n}\right)\right\}=\{0\}
$$

as described in section 2.2. Now the nodal foree vector $\{F\}$ depends on the values of the scalar electric potential $\Phi$ and the normal component of the electric ficld $E_{n}$.

In order to illustrate the procedure of the $\mathrm{FE} / \mathrm{BL}-\mathrm{Method}$ a capacitive acceleration sensor as shown in Fig. 1.5 is used. Finite elements are used to describe the mechanical field in the whole structure, whereas the electric field in the gap between the two electrodes is modeled by boundary clements. This approach has the advantage, that deformations of electrode 2 will not cause a deformation of surrounding finite clements which would be necessary to describe the clectric field in the case of a pure FE-modeling. The two boundary element matrices $\mathbf{H}_{\phi}$ and $\mathbf{G}_{\phi}$ have to be updated corresponding to the mechanical displacement. The direct coupling of (1.9) and (1.10) leads to a nonlinear system of equations. Using predictor values for the calculation of the electrostatic force, a decoupling into an electric and a mechanical matrix equation can be achicved. To ensure the strong coupling between the electric and mechanical quantities a Predictor/Multicorrector Algorithm as described in [9] has been successfully used. 


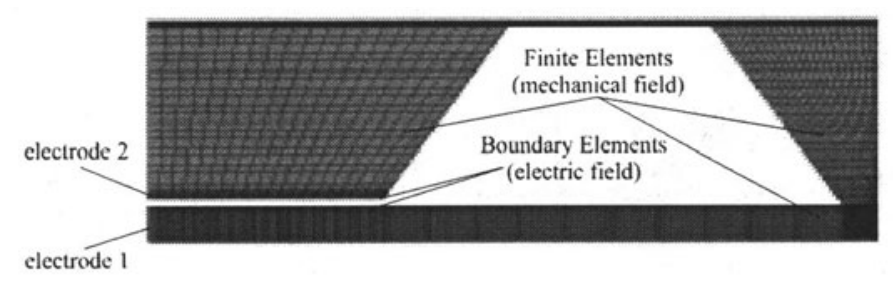

Figlire 5 FEM/BEM discretization of a capacitive acceleration sensor

\subsection{ACCELERATION SENSOR}

The capacitive acceleration sensor (Fig. 1.5) is fabricated by micromachined techniques. Loading the sensor with an acceleration step causes the silicon structure to be deformed (Fig.

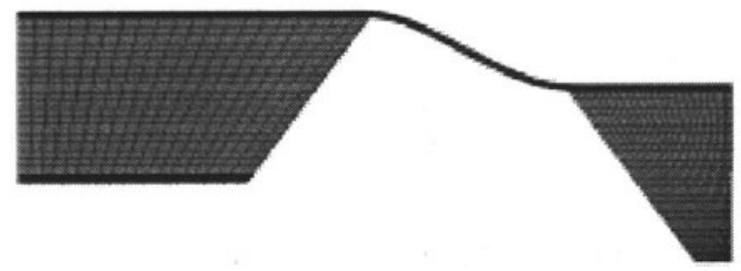

Figure 6 Deformations of the moving part of the acceleration sensor due to an acceleration pulse

1.6) and the change in the capacitance is a direct measure of the acceleration. Without using any controller, the silicon structure oscillates with its eigenfrequency to a new position according to the acceleration step (Fig. 1.7). Applying a PID-controlled voltage to the electrodes, the transient response can be kept to a minimum and the silicon structure moves to the old position (Fig. 1.7) and the controller output voltage (which is applyed to the electrodes) is now a direct measure of the acceleration.

\subsection{MICROPUMP}

A typical microsystem is a micromachined pump, shown in Fig. 1.8. If an electric voltage is applied to the electrodes, the elastic pump diaphragm (electrode 2) is deformed by the electrostatic force and bends towards the counterelectrode (electrode 1). Thereby, fluid will be sucked in through the inlet valve. When the supply voltage is switched off, the relaxation of the diaphragm will push the fluid through the outlet valve. The FE.M/BEM discretization of the actuation unit (Fig. 1.8) is performed according to Fig. 1.9. Finite elements are used to describe the mechanical field in the two electrodes, whereas the electric field in the gap between is modeled by boundary elements. This approach has the advantage, that the elastic pump diaphragm (electrode 2) can move towards the stationary counterelectrode (electrode 1) without deforming any finite element which would otherwise (with pure FE-modeling) be necessary to describe the electric ficld. 


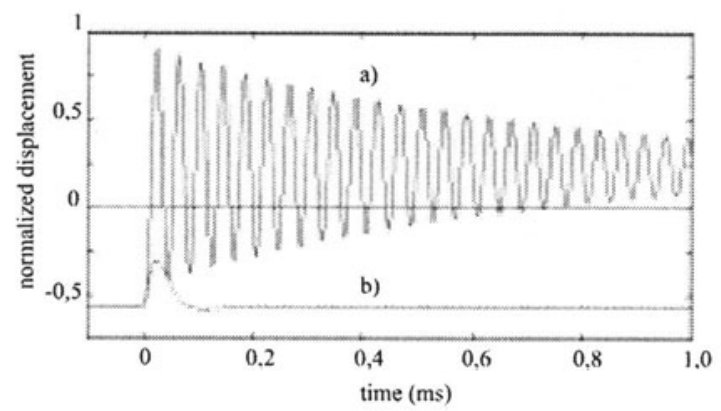

Higure 7 a) Uncontrolled b) controlled dynamic behaviour due to an acceleration step.

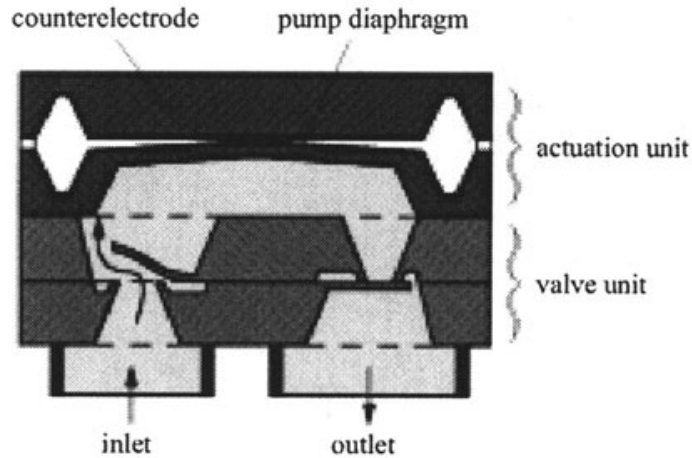

Figure \& Schematic view of an electrostatically driven micropump

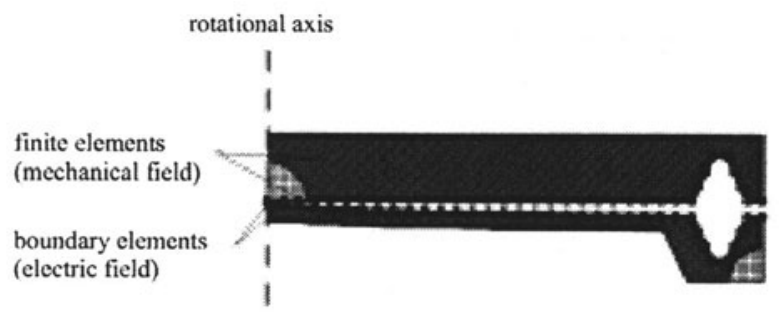

Figure $9 \mathrm{FEM} / \mathrm{BEM}$ discretization of the actuation unit

This pump has a diameter of $7 \mathrm{~mm}$, a total height of about $1 \mathrm{~mm}$ and a gap thickness of $4 \mu \mathrm{m}$ between the elastic pump diaphragm and the counterelectrode. Fig. 1.9 shows the mechanical deformations of the actuation unit when a de voltage is applied. One point of investigation was the nonlinear dynamic response of the micropump. The pump was excited by a sinusoidal voltage with a frequency of $1 \mathrm{kHz}$ and different amplitudes. The dynamic behaviour was analyzed by 


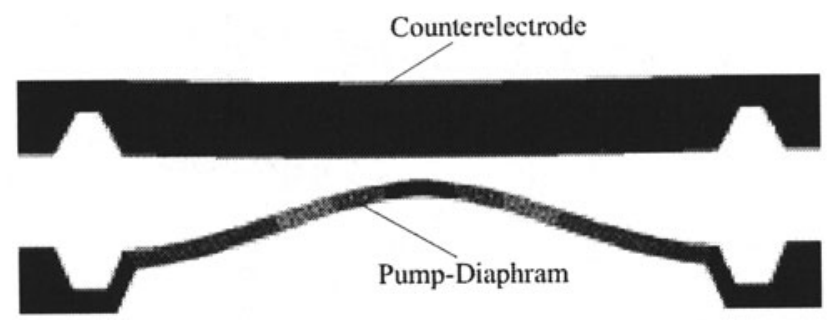

computing the electrostatic-mechanical system with the described calculation scheme. In Fig. 1.10 , the center displacement of the clastic pump diaphragm is depicted as a function of the applicd voltage. The corresponding frequency spectra can be seen in Fig. 1.11. For a good comparability each curve in Fig. 1.10 and Fig. 1.11 is normalized to its maximum.

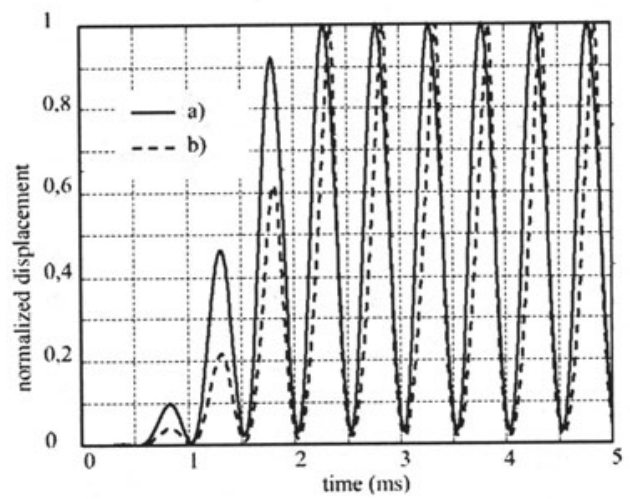

Figure 10 Mechanical displacement in the center of the pump diaphragm. a) Original amplitude of applied voltage. b) Twice the original amplitude of applied voltage

\section{MAGNETOMECHANICAL TRANSDUCERS 4.1 BASIC EQUATIONS}

For the quasi-stationary case (neglecting displacement currents) the magnetic field is computed by solving Maxwells cquations

$$
\begin{aligned}
\nabla \times \vec{H} & =\vec{J} \\
\nabla \times \vec{E} & =-\frac{\partial \vec{B}}{\partial t} \\
\vec{B} & =\mu \vec{H} \\
\vec{J} & =\gamma \vec{E} .
\end{aligned}
$$

In (1.11) - (1.14) $\vec{H}$ denotes the magnetic field intensity, $\vec{B}$ the magnetic flux density, $\vec{J}$ the current density, $\vec{E}$ the electric field intensity. $\mu$ the permeability and $\gamma$ the electric conductivity. By introducing the magnetic vector potential $\vec{A}$ as follows

$$
\vec{B}=\nabla \times \vec{A}
$$




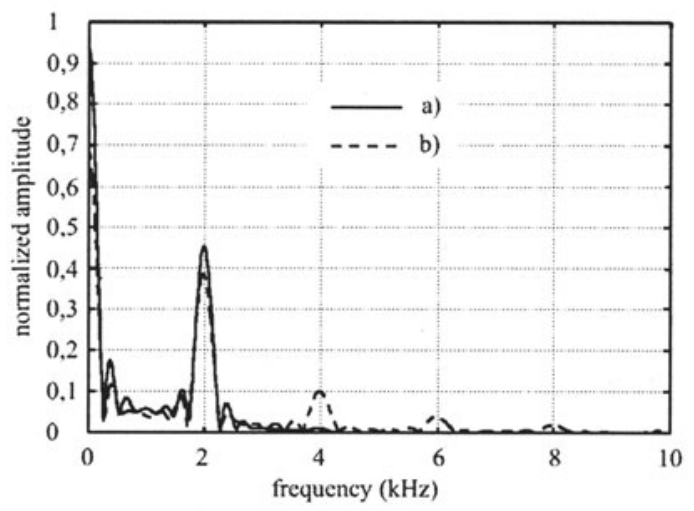

Figure 11 Frequency spectrum of the mechanical displacement in the center of the clastic pump diaphragm. a) Original amplitude of applied voltage. b) Twice the original amplitude of applicd voltage

and applying the Coulomb gauging according to [10], the following matrix equation is derived

$$
\left(\begin{array}{cc}
\mathbf{M}_{A \Lambda} & \mathbf{M}_{A \dot{ }} \\
\mathbf{M}_{A \phi}^{T} & \mathbf{M}_{\phi \dot{\phi}}
\end{array}\right)\left(\begin{array}{c}
\{\dot{A}\} \\
\{\dot{\Phi}\}
\end{array}\right)+\left(\begin{array}{cc}
\mathbf{P}_{A A} & 0 \\
0 & 0
\end{array}\right)\left(\begin{array}{c}
\{. A\} \\
\{\Phi\}
\end{array}\right)=\left(\begin{array}{c}
\{Q\} \\
\{0\}
\end{array}\right) .
$$

Herein, $\mathbf{M}_{A A}$ and $\mathbf{M}_{\phi \phi}$ denote the magnetic mass matrix of the magnetic vector potential $\vec{A}$ and scalar electric potential $\Phi, \mathbf{M}_{A \Phi}$ the coupling matrix between them, $\mathbf{P}_{A A}$ the magnetic stiffness matrix and $\{Q\}$ the nodal vector corresponding to prescribed electric currents. In the case of a voltage loaded coil this equation has to be solved together with the electric circuit equation [11], which determines the current in the coil.

The first coupling term is given by the movement of a coil or conductive body (with velocity $\vec{v})$ in a magnetic field $\vec{B}$. Therewith, an additional current density $\vec{J}_{v}$ is induced in the coil

$$
\vec{J}_{v}=\gamma \vec{v} \times \vec{B}=\gamma \frac{\partial \vec{u}}{\partial t} \times \vec{B}
$$

which has to be added to the electric circuit equation.

Furthermore, the magnetic volume force, resulting from the interaction between the total magnetic field $\vec{B}$ and coil current density $\vec{J}$, expressed by

$$
\vec{f}_{V}=\vec{J} \times \vec{B}
$$

has to be added to the nodal force vector of the mechanical equation.

The direct coupling of the magnetic and mechanical matrix equation leads to a nonlinear and unsymmetric system of equations. Using predictor values for the calculation of the magnetic volume force and the mechanical velocity $\vec{v}$ of the coil, a decoupling into a magnetic and mechanical matrix equation can be achieved. To ensure the strong coupling between the magnetic and mechanical quantities a Predictor/Multicorrector Algorithm as described in [12] is used. 


\subsection{ELECTROMAGNETIC ACOUSTIC TRANSDUCER (EMAT)}

A typical setup of an EMAT as used in nondestructive material testing is shown in Fig. 1.12. It can be used either to generate (transmitting mode) or to detect (receiving mode) lamb waves in the material under test [13]

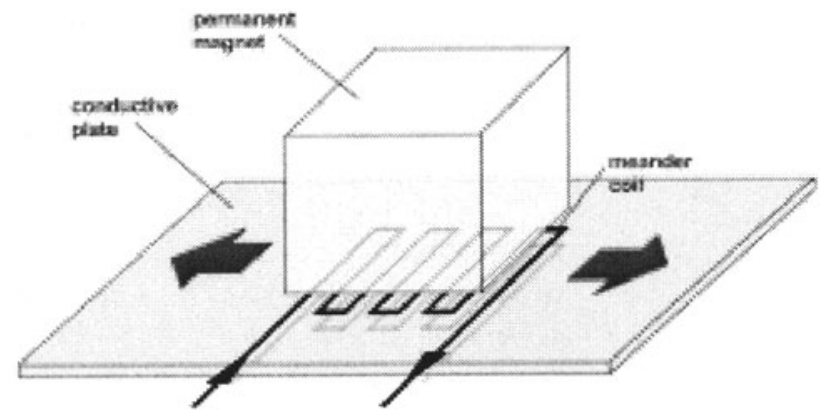

Figure 12 Setup of an electromagnetic acoustic transducer ( $\mathrm{EM} \Lambda \mathrm{T})$

- Transmitting mode: Loading the coil by a short tone burst signal, the time varying magnetic field induces eddy currents in the conductive matcrial under test. The penetration depth of these eddy currents is given by the classical skin depth $\delta$

$$
\delta=\frac{1}{\sqrt{\pi f \gamma \mu}}
$$

where $f$ denotes the frequency of the time varying magnetic field, $\gamma$ the electrical conductivity and $\mu$ the magnetic permeability of the material under test. The interaction of these eddy currents and the overall magnetic field of the permanent magnet results in a lamb wave propagating in the conductive shect.

- Receiving mode: When the lamb wave passes the region of the receiving EMAT, which is subjected to the static magnetic field of the permanent magnet, locally eddy currents are induced in the conductive metallic sheet. Therewith. the time varying magnetic field of these eddy currents induces a voltage in the meander coil.

The main drawback of conventional FE-discretization is the high solution time due to the large number of unknowns, especially in the $3 \mathrm{D}$ case. One reason for the high demands on computer resources is that the generated magnetic FL mesh, describing the magnetic field in the penetration depth $\delta$, which is typically $50 \mu \mathrm{m}$ deep, has to be extremely fine. Using conventional FE-discretization the mechanical ficld is also solved at this very fine mesh, which is only necessary for the magnetic ficld problem.

For this problem a new multigrid approach was utilized which reduces the solution time considerably. This was achieved by using a fine $\mathrm{FL}$ grid for the magnetic field and a much coarser one for the mechanical problem (see Fig. 1.13) and on the other hand by applying very fast multigrid methods to solve the linear equation systems at each time step of the transient analysis [14].

For a precise 2D model, the FE-discretization results in a total number of about 300.000 unknowns. A computer run for 200 time steps with direct conventional solution strategies needs 


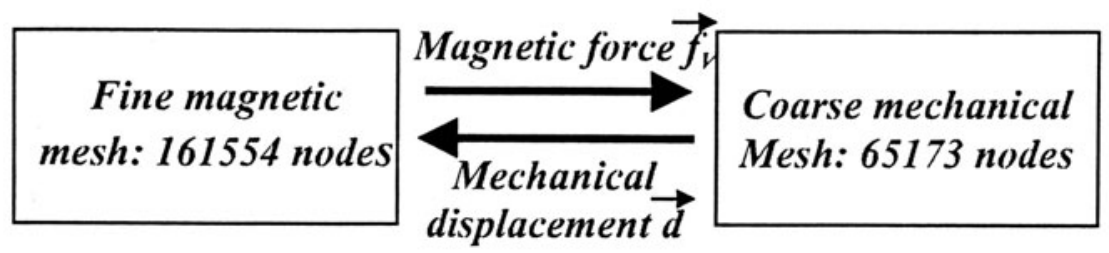

Figure 13 FE grids for the magnetic and mechanical problem

approximately 21 hours on a SGI, Octan $195 \mathrm{MHz}$. Using multigrid solution techniques the solution time can be reduced to 50 minutes. In the 3D case the advantages of the presented multigrid approach in comparison with standard solvers are even higher.

To show the applicability of the developed calculation scheme for the transient behavior of the EMAT in the transmitting mode as well as in the receiving mode, a set of 2D and 3D simulations has been carried out and compared with experimental data. Fig. 1.14 a) shows the dispersion curves of the group velocity for various lamb modes and lig. 1.14 b) compares the measured and simulated induced voltage in the meander coil of an E.MAT in receiving mode. In both cases a very good agreement between measurement and simulation could be obtained.

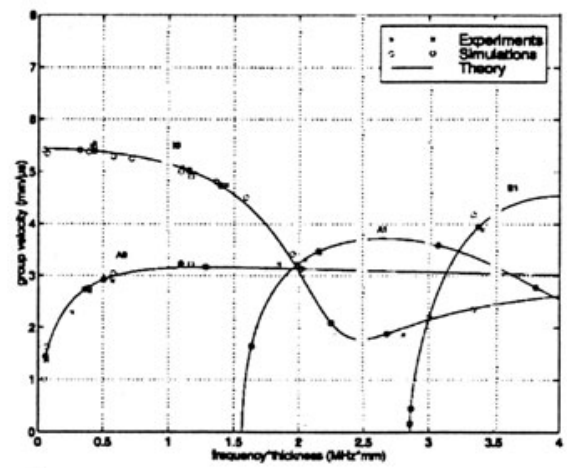

a.)

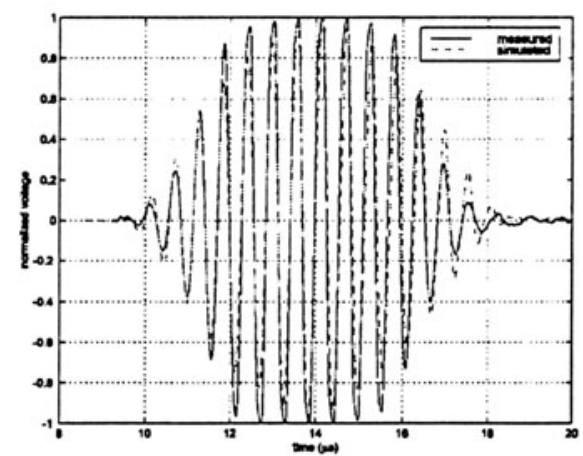

b.)

Figure 14 a) Group velocity curves for A0. A1, SO and S1 lamb wave modes in aluminium plates. b) Normalized induced voltage in the coil of the receiving EMAT

\section{REFERENCES}

[1] Lerch, R.; Landes, H.; Kaltenbacher, M.: CAPA User Mamual . Tech. Rept. 3.3. University of Linz, Altenberger Str. 69, A-4040 Linz, 1999.

[2] Mason, W. P. (Ed.): Physical Acoustics, Vol. I - Part A, London: Academic Press, 1964 
[3] Lerch, R.: Simulations of Piezoelectric Devices by' Two- and Three-Dimensional Finite Elements. IEEE Trans. on Ultras., Ferroel. and Freq. Control, Vol. 37, No. 3, 1990, pp. 233-247

[4] Lerch, R.: Finite Element Analysis of Piezoelectric Transducers, Proc. IEEE Ultrasonics Symposium, 1988. pp. 643-654

[5] Hughes, T. J. R.: The Finite Element Method, New Jersey: Prentice-Hall, 1987

[6] Lerch, R.: Landes, H.; Kaarmann, H.: Finite Element Modeling of the Pulse-Echo Behavior of Ultrasound Transducers, Proc. IEEL Ultrasonics Symposium, 1994, pp. 1021-1025

[7] Liccardt,P.; Niederer, K; Scheiter, T.; Hicrold, C.: Surface micromachined ultrasound transducer in CMOS technology, Proc. IEEE Ultrasonics Symposium, 1996, pp. 959-962

[8] Kaltenbacher, M.; Landes, H.; Niederer, K; Lerch, R.: 3D Simulation of Controlled Micromachined Capacitive Ultrasound Transducers, Proc. IEEE Ultrasonics Symposium, 1999 (to appear)

[9] Kaltenbacher, M.; Landes, H.; Lerch, R.: A Finite Element / Boundary-Element Method for the Simulation of Coupled Electrostatic-Mechanical Systems, J. Phys. III France 7, 1997, pp. 1975-1982

[10] Biro, O.; Preis, K.: On the Use of Magnetic Vector Potential in the Finite Element Analysis of Three-Dimensional Eddy Currents, IEEE Trans. on Magnetics, Vol. 25, No. 4, July 1989, pp. 3145-3159

[11] Lconard, P.J.; Rodger, D.: Voltuge forced coils for 3D finitc-element electromagnetic models, IEEE Transactions on Magnetics, Vol. 24, No,6, November 1988 pp. 2579-2581

[12] Kaltenbacher, M.; Landes, H.; Lerch, R.: A Strong Coupling Model for the Sinulation of Magnetomechanical Systems using a Predictor/Multicorrector Algorithm, Applied Computational Electromagnetics Society Journal, 1997, Vol. 12, No,2. pp. 102-106

[13] M. Kaltenbacher, R. Lerch, H. Landes, K. Ettinger, B. Tittmann, Computer Optimization of Electromagnetic Acoustic Transducers, Proc. IEEE Ultrasonics Symposium, 1999, pp. 1029-1034

[14] M. Jung, U. Langer, Applications of multilevel methods to practical prohlems, Surv. Math. Ind. 1,1991 